\title{
Ostéonécrose avasculaire des maxillaires après traitement d'un myélome multiple par bisphosphonates. A propos d'un cas
}

\author{
Jaws avascular osteonecrosis after treatment of multiple myeloma \\ with bisphosphonates. Report of a case
}

\author{
ABDELKADER BENNANI ${ }^{1}$, ABDELILAH OUJILAL ${ }^{2}$, SALIHA CHBICHEB ${ }^{1}$, BOUCHRA TALEB ${ }^{1}$, WAFAE EL WADY ${ }^{1}$
}

\begin{abstract}
RÉSUMÉ
L'ostéonécrose avasculaire des maxillaires sous bisphosphonates est assez souvent rapportée lors du traitement du myélome multiple. Actuellement, le nombre des cas d'ostéonécroses décrites dans la littérature avoisine 1500.

Monsieur A.L, âgé de 62 ans, est traité pour un myélome multiple à lgG de stade Illa par bisphosphonates intraveineux associés à une chimiothérapie et à une corticothérapie. Ce patient a développé une ostéonécrose maxillo-mandibulaire droite, après des extractions dentaires. Le bilan radiologique a découvert une ostéolyse étendue dans la région postérieure droite de la branche horizontale, de l'angle et du ramus mandibulaires. Ce patient a bénéficié d'une ablation, sous anesthésie générale, des esquilles et des séquestres osseux maxillaires mobiles associés à un traitement antiseptique et antibiotique et à une oxygénothérapie hyperbare. L'ostéonécrose des maxillaires sous bisphosphonates touche les patients âgés de 61 ans en moyenne, de race blanche, et siège avec prédilection dans la région mandibulaire postérieure. Les traitements avec le pamidronate ou l'acide zolédronique, l'extraction dentaire antérieure, l'âge avancé du patient, la posologie et la durée d'exposition aux bisphosphonates concourent au développement des ostéonécroses. Actuellement, le traitement préconisé pour ces ostéonécroses est essentiellement symptomatique et conservateur. Le traitement préventif consiste en une mise en état bucco-dentaire avant le début du traitement par les bisphosphonates. (Med Buccale Chir Buccale 2009 ; 15: 39-44).
\end{abstract}

mots clés: bisphosphonates, myélome multiple, ostéonécrose, mâchoire

\section{SUMMARY}

Bisphosphonates-induced avascular osteonecrosis of the jaws is often reported after treatment of multiple myeloma. Currently, the number of cases of this osteonecrosis described in the literature is approximately 1500.

Mr. A.L of 62 years old and treated for a multiple myeloma IgG stage Illa by intravenous bisphosphonates associated to chemotherapy and corticotherapy. This patient developed an osteonecrosis of the jaws following teeth avulsions. The radiological exam discovered a large osteolyse of the right posterior region of mandible. This patient treated by an ablation, under general anesthesia, of bone sequestrum associated to an antiseptic and antibiotic treatment and a hyperbaric oxygen treatment. 
médecine buccale chirurgie buccale

VOL. $15, \mathrm{~N}^{\circ} 1$ 2009

page 40

Bisphosphonates-induced osteonecrosis of the jaws affect patients aged of 61 years old of white race and seat with predilection in posterior region of mandible. Treatments by pamidronate or zoledronic acid, previous dental extraction, advanced age of the patient, posology and the length of exposition to bisphosphonates have been implied in the development of this osteonecrosis. Currently, the recommended treatment for this osteonecrosis is essentially symptomatic and conservative. The preventive treatment consists on a complete dental evaluation performed before commencing bisphosphonate treatment. (Med Buccale Chir Buccale 2009 ; 15: 39-44).

key words: bisphosphonates, multiple myeloma, osteonecrosis, jaw

L'ostéonécrose des maxillaires peut être liée à de nombreuses étiologies dont les plus fréquentes sont représentées par la radiothérapie et certains traitements médicamenteux. Parmi les médicaments incriminés dans ces ostéonécroses, on retrouve le plus souvent les bisphosphonates (BP) lors du traitement du myélome multiple. En effet, les premiers cas ont été rapportés en 2003 et actuellement le nombre de cas d'ostéonécrose des maxillaires sous BP décrits dans la littérature avoisine 1500 cas $^{[1-3]}$. Cette entité pathologique pose actuellement encore des problèmes, en particulier étiopathogéniques et thérapeutiques. Nous rapportons un nouveau cas d'ostéonécrose maxillo-mandibulaire chez un patient atteint de myélome multiple traité par BP. A travers ce cas clinique, nous allons discuter l'épidémiologie, le diagnostic ainsi que le traitement de ces ostéonécroses.

\section{OBSERVATION CLINIQUE}

Monsieur A.L, âgé de 62 ans, a consulté dans le service en septembre 2007 pour des douleurs et un retard de cicatrisation gingivale après des extractions dentaires multiples maxillo-mandibulaires réalisées 1 an auparavant.

L'interrogatoire du patient a montré qu'il avait un myélome multiple à lgG de stade IIla depuis mai 2000, traité par radiothérapie, chimiothérapie (Melphalan), dexaméthasone, 5 cures mensuelles de pamidronate puis 13 cures mensuelles d'acide zolédronique. En septembre 2006, 46, 47 et 48 ont été extraites en raison d'une douleur mandibulaire postérieure droite. Les suites opératoires ont été marquées par l'apparition d'une tuméfaction mandibulaire douloureuse et surinfectée, en regard des foyers d'extraction, ne cédant pas au traitement antiseptique et antibiotique. Le bilan radiologique a montré une perte de substance osseuse avec fracture mandibulaire pathologique, ce qui a motivé la réalisation d'un curetage mandibulaire postérieur droit sous anesthésie générale, associé à une ostéosynthèse par mini-plaque. L'examen anatomopathologique du tissu cureté a précisé qu'il s'agissait d'une ostéite colonisée par des Actinomyces, sans signe de malignité. Un traitement antiseptique et antibiotique par amoxicilline a été prescrit pendant 50 jours. Après 4 mois, le cliché panoramique a montré la persistance du foyer d'ostéite mandibulaire. Ceci a motivé la réalisation, en mai 2007, d'un deuxième curetage mandibulaire avec dépose de la mini-plaque d'ostéosynthèse. A son arrivée dans le service en septembre 2007, l'examen clinique exobuccal a montré une fistule cutanée en regard du foyer d'ostéite qui drainait un exsudat purulent, associé à une tuméfaction génienne droite dure et douloureuse avec une hypoesthésie labio-mentonnière homolatérale (Fig.1).

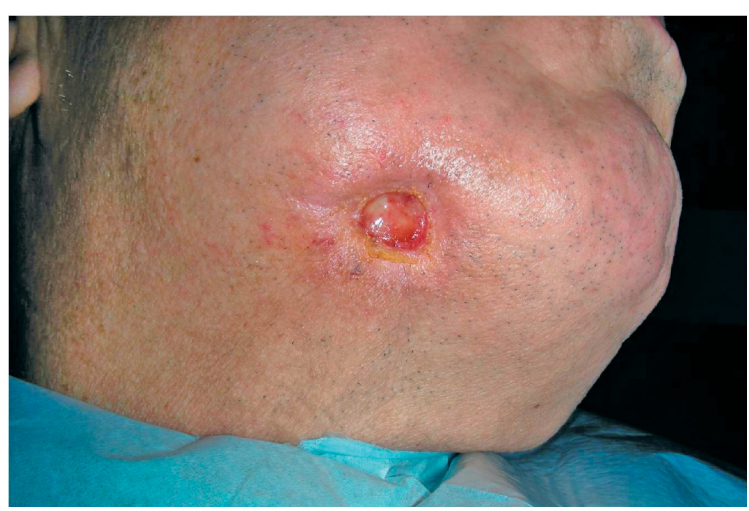

Figure 1 : Fistule cutanée siégeant dans la région submandibulaire droite.

Cutaneous fistula in the right submandibular region. 

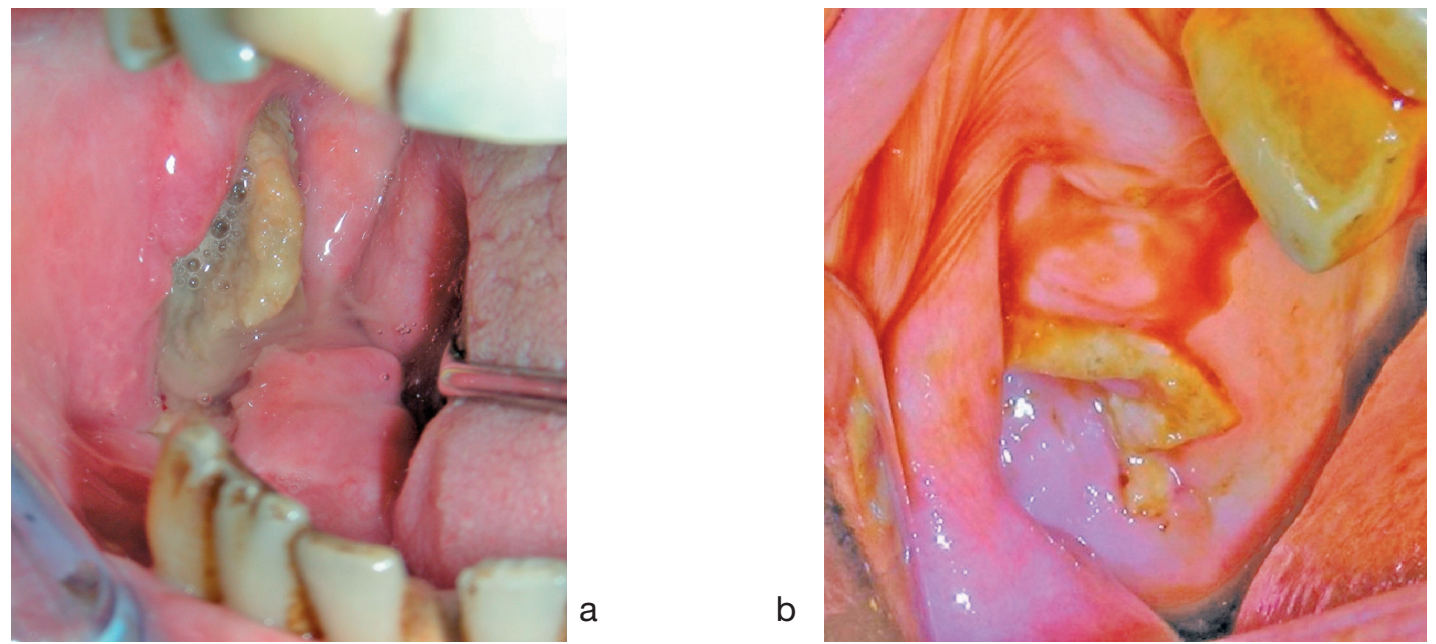

Figure 2 : Exposition d'un tissu osseux nécrosé et surinfecté intéressant les deux maxillaires (2a : mandibule ; $2 \mathrm{~b}$ : maxillaire).

Necrosis and bone infection of both maxillary (2a: mandible; 2b: maxilla).

A l'examen endobuccal, on notait l'absence de 18, $17,16,15,24,25,27,28,44,45,46,47$ et 48 et deux ulcérations muqueuses avec exposition osseuse intéressant le maxillaire et la mandibule dans la région postérieure droite (Fig. 2a et 2b).

Le bilan radiologique (radiographie panoramique et TDM du massif facial) a montré une ostéolyse étendue dans la région postérieure droite de la branche horizontale, de l'angle et du ramus mandibulaires, un retard de cicatrisation sur les alvéoles d'extraction maxillaires postérieures droites et une alvéolyse généralisée (Fig.3a et 3b). Ce patient a bénéficié d'une ablation, sous anesthésie générale, des esquilles et des séquestres osseux mobiles des

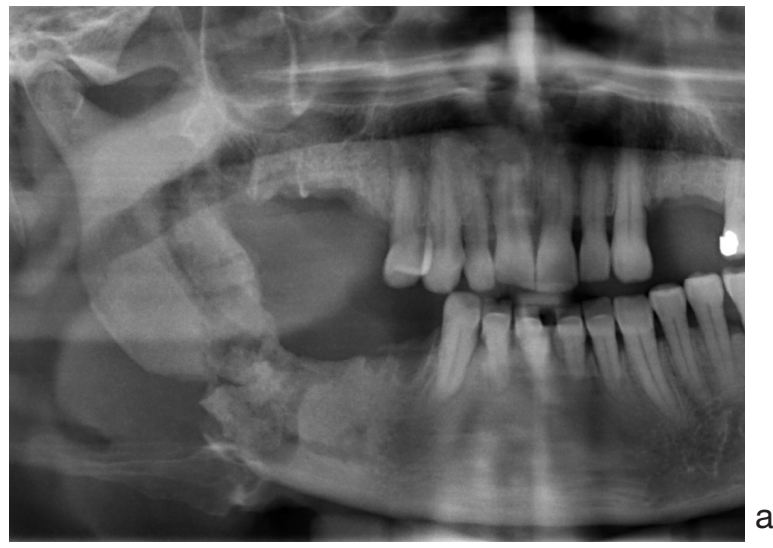

a

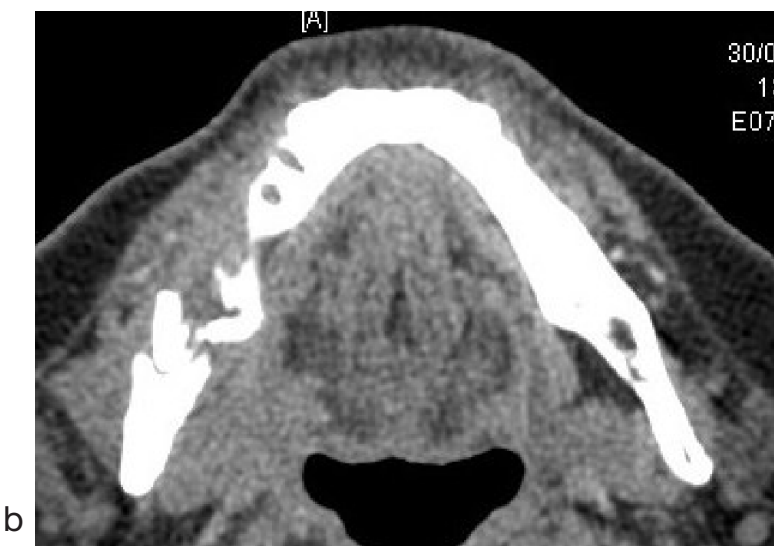

Figure 3 : Radiographies préopératoires (3a : panoramique dentaire, 3b : coupe axiale TDM) montrant l'image d'un séquestre osseux mandibulaire droit.

Preoperative X-rays (3a: Dental panoramic, 3b: CT axial) showing a bone sequester in the right mandibular region. une perte de substance osseuse étendue dans la région mandibulaire droite postérieure et une bonne cicatrisation osseuse maxillaire (Fig. 5). Le patient est décédé en décembre 2007 d'une atteinte pulmonaire sévère, secondaire à son myélome. deux os maxillaires. L'examen anatomopatholoosseuse sans signe histologique de malignité nolone/métronidazole) et une oxygénothérapie hyperbare (20 séances) ont été effectués.

Le contrôle radiologique, un mois après, a montré médecine

buccale

chirurgie

buccale

VOL. $15, N^{\circ} 1$

2009

page 41 


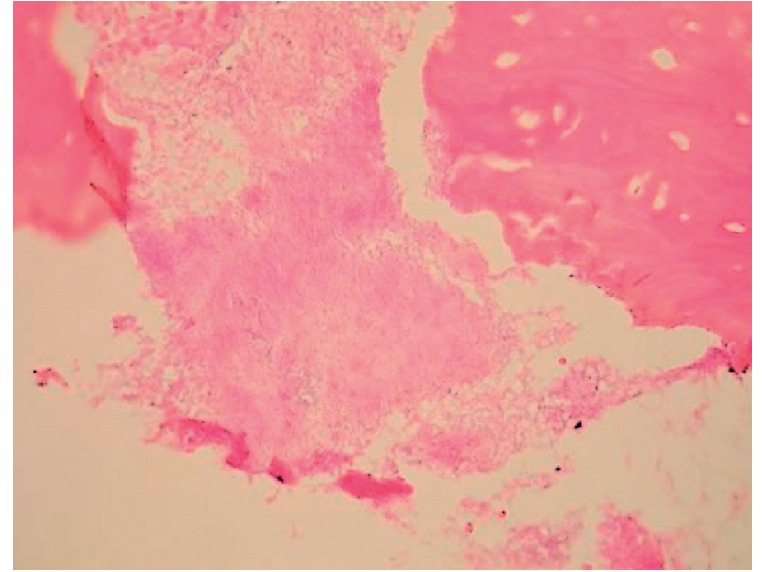

Figure 4 : Coupe histopathologique montrant une nécrose osseuse (cavités ostéocytaires vides) et une surinfection actinomycosique (HES, x 200).

Histopathological picture showing bone necrosis (osteocytes empty cavities) and actinomycosis infection (HES, $x$ 200).

médecine

buccale

chirurgie

buccale

VOL. $15, \mathrm{~N}^{\circ} 1$

2009

page 42

\section{DISCUSSION}

Les BP et en particulier les aminobisphosphonates sont des analogues des pyrophosphates, indiqués dans le traitement de plusieurs affections dont le myélome multiple ${ }^{[4]}$. Cependant, ces BP sont à l'origine de nombreuses complications, dont l'ostéonécrose avasculaire des maxillaires [4]. L'étiopathogénie des ostéonécroses induites par les BP semble être liée à la susceptibilité des maxillaires par rapport aux autres os du squelette, à l'activité anti-ostéoclastique et anti-angiogenique des BP, au non couplement de l'équilibre ostéoclaste-ostéoblaste et à l'effet « bandwagon » qui augmente la susceptibilité des maxillaires à l'osteonécrose ${ }^{[1]}$. Cette complication est trouvée, le plus fréquemment, chez les patients atteints de myélome multiple, âgés de 61 ans en moyenne, de race blanche. Elle siège avec prédilection dans la région mandibulaire postérieure [3,5-8].

Parmi les facteurs de risque de développement d'une ostéonécrose lors du traitement du myélome multiple, on retrouve essentiellement les traitements utilisant le pamidronate ou l'acide zolédronique, l'extraction d'une dent, l'âge avancé du patient au moment du diagnostic ainsi que la

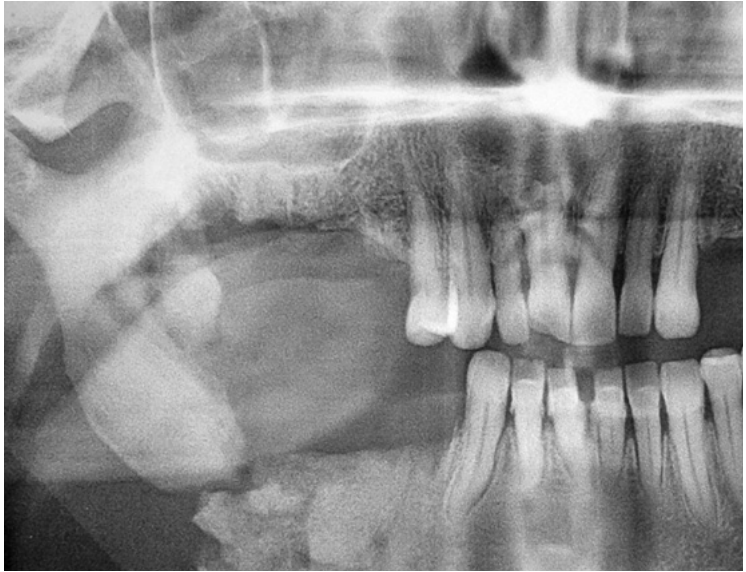

Figure 5 : Cliché panoramique de contrôle réalisé un mois après la $2^{\mathrm{e}}$ intervention.

Dental panoramic of control carried out one month after the 2nd intervention. posologie et une durée d'exposition aux BP supérieure à 2,5 ans $[3,7,8]$. Les autres traitements, à savoir la chimiothérapie et la corticothérapie, pourraient également jouer un rôle dans la survenue de ces ostéonécroses ${ }^{[3]}$.

Cliniquement, l'ostéonécrose des maxillaires se manifeste par une exposition osseuse, qui peut être associée selon les cas à une tuméfaction génienne ou mandibulaire secondaire à la réaction inflammatoire liée au foyer d'ostéite, une douleur ou une mobilité dentaire. L'évolution de l'ostéonécrose peut s'accompagner de l'apparition d'un séquestre osseux important, une communication bucco-nasale ou bucco-sinusienne, une fistule cutanée ou une fracture mandibulaire pathologique ${ }^{[4,5]}$.

Les signes radiologiques sont très discrets, se traduisant généralement par un remaniement osseux modéré, un élargissement de l'espace desmodontal ou par la persistance de l'alvéole après extraction dentaire ${ }^{[2,3]}$. A un stade avancé, l'ostéonécrose se présente comme une image radioclaire mal définie, associée ou non à un séquestre osseux radiopaque. Cet aspect radiologique est non spécifique et doit être différencié d'une ostéomyélite chronique, d'une ostéoradionécrose infectée, d'une pathologie tumorale 
osseuse primaire (myélome) ou métastatique et d'une fracture pathologique des maxillaires [2]. L'examen histopathologique montre une nécrose osseuse associée à une surinfection bactérienne et du tissu de granulation [2]. Le diagnostic d'actinomycose doit être corrélé avec les résultats de la culture bactérienne ${ }^{[2]}$. Dans certains cas, si une métastase osseuse tumorale est suspectée, une biopsie osseuse doit être réalisée.

Bedogni et coll. [9] ont trouvé une corrélation entre l'examen histopathologique, et la TDM et I'IRM qui sont les examens radiographiques de choix pour l'évaluation de l'ostéonécrose induite par les BP. La TDM montre l'augmentation de la densité osseuse, une réaction périostée et un séquestre osseux dans les stades avancés. Cependant, les altérations osseuses sur l'IRM varient entre les régions osseuses exposées ou non exposées à la cavité buccale. Ainsi, dans les régions exposées, l'IRM montre un signal faible en T1 et T2 qui correspond histologiquement à l'absence de cellules et de vaisseaux caractéristique de l'ostéonécrose. Tandis que dans les régions non exposées, I'IRM présente une hypodensité en T1 et une hyperdensité en T2 et en IR qui correspondent histologiquement à une hypercellularité, une ostéogenèse et une hypervascularisation caractéristiques mais non exclusives de l'ostéomyélite ${ }^{[9]}$.

Actuellement, le traitement préconisé pour l'ostéonécrose est essentiellement symptomatique et conservateur. II consiste à réaliser des rinçages buccaux antiseptiques (chlorhexidine aqueuse $0,1 \%$ ), une antibiothérapie en cas de surinfection locale, d'abcès ou de fistulisation et un débridement chirurgical de l'os nécrosé à type d'ablation des esquilles ou des séquestres osseux mobiles ${ }^{[4]}$.
Dans certains cas d'ostéonécrose étendue, seul un traitement agressif (résection interruptrice de la mandibule...) permet d'obtenir la résolution de la douleur et l'infection aiguë [2].

D'après Ruggiero et Marx ${ }^{[2,5,6]}$ une résection segmentaire associée à une reconstruction immédiate par une plaque d'ostéosynthèse est nécessaire lors d'une fracture mandibulaire pathologique. Par contre, la reconstruction mandibulaire immédiate avec un greffon osseux vascularisé ou non ne peut pas être envisagée car il existe un risque de nécrose osseuse sur le site receveur en raison de l'effet systémique des $\mathrm{BP}$ [2].

L'oxygénothérapie hyperbare pour le traitement de l'ostéonécrose reste controversée car, pour certains ${ }^{[2,5,6]}$ elle n'arrive pas à limiter la progression de l'ostéonécrose alors que, pour Freiberger et coll. [10], elle entraine une diminution de la taille et du nombre des foyers d'ostéonécrose et une cicatrisation plus rapide à l'arrêt de l'administration des BP. Cependant, en raison de l'efficacité oncologique incontestable des BP et de leur longue demi-vie osseuse allant jusqu'à plusieurs années, il ne semble pas justifié d'arrêter le traitement par BP [2,4-6].

Les interventions chirurgicales dento-alvéolaires électives comme les extractions, les implants dentaires ou les interventions de chirurgie endodontique, doivent être évitées parce qu'elles peuvent favoriser le développement d'osteonécrose [2]. Ainsi, le meilleur traitement à préconiser avant le début du traitement des patients atteints de myélome multiple par des BP est la prévention en réalisant une mise en état bucco-dentaire et en informant les patients du risque et des moyens de prévention de l'ostéonécrose des maxillaires. médecine

buccale chirurgie buccale

VOL. $15, \mathrm{~N}^{\circ} 1$ 2009 page 43 


\section{RÉFÉRENCES}

1 - Sarin J, DeRossi SS, Akintoye SO. Updates on bisphosphonates and potential pathobiology of bisphosphonate-induced jaw ostéonecroses. Oral Dis 2008 ; $14: 277-85$.

2 - Ruggiero S L, Fantasia J, Carlson E. Bisphosphonaterelated osteonecrosis of the jaw: background and guidelines for diagnosis, staging and management. Oral Surg Oral Med Oral Pathol Oral Radiol Endod 2006 ; $102: 433-41$

3 - Boonyapakorn T, Schirmer I, Reichart PA, Sturm I, Massenkei G. Bisphosphonate-induced osteonecrosis of the jaws: prospective study of 80 patients with multiple myeloma and other malignancies. Oral Oncol $2008 ; 44: 857-69$.

4 - Lobato J, Mauricio AC, Rodrigues JM, Cavaleiro MV, Cortez PP, Xavier L, Botelho C, Hussain NS, Santos JD. Jaw avascular osteonecrosis after treatment of multiple myeloma with zoledronate. J Plast Reconstr Aesthet Surg $2008 ; 61: 99-106$.

5 - Marx R, Sawatari Y, Fortin M, Brounmand V. Biphosphonate-induced exposed bone (osteonecrosis/osteopetrosis) of the jaws: risk factors, recognition, prevention, and treatment. J Oral Maxillofac Surg 2005; $63: 1567-75$

6 - Ruggiero S L, Mehrotra B, Rosenberg T J, Engroff S L. Osteonecrosis of the jaws associated with the use of biphosphonate: A review of 63 cases. J Oral Maxillofac Surg $2004 ; 62$ : 527-34.
7 - Badros A, Weikel D, Salama A, Goloubeva O, Schneider A, Rapoport A, Fenton R, Gahres N, Sausville E, Ord R, Meiller T. Osteonecrosis of the jaw in multiple myeloma patients: clinical features and risk factors. J Clin Oncol 2006 ; 24 : 945-52.

8 - Bamias A, Kastritis E, Bamia C. Osteonecrosis of the jaw in cancer after treatment with bisphosphonates: incidence and risk factors. J Clin Oncol 2005 ; 23 : 8580-7.

9 - Bedogni A, Blandamura S, Lokmic Z, Palumbo C, Ragazzo M, Ferrari, F Tregnaghi A, Pietrogrande F, Procopio O, Saia G, Ferretti M, Bedogni G, Chiarini L, Ferronato G, Ninfo V, Lo Russo L, Lo Muzio L, Nocini PF. Bisphosphonate-associated jawbone osteonecrosis: a correlation between imaging techniques and histopathology. Oral Surg Oral Med Oral Pathol Oral Radiol Endod 2008 ; 105 : 358-64.

10 - Freiberger J, Padilla-Burgos R, Chhoeu A, Kraft KH, Boneta O, Moon RE, Piantadosi CA. Hyperbaric oxygen treatment and biphosphonate-induced osteonecrosis of the jaw: a case series. J Oral Maxillofac Surg $2007 ; 65: 1321-27$.
VOL. $15, \mathrm{~N}^{\circ} 1$

2009

page 44 buccale

chirurgie

buccale 